\title{
DNLS with Impurities
}

\author{
Jesús Cuevas $^{1}$ and Faustino Palmero ${ }^{2}$ \\ ${ }^{1}$ Grupo de Física No Lineal, Departamento de Física Aplicada I, Escuela \\ Universitaria Politécnica. Universidad de Sevilla. C/ Virgen de África, 7, 41011 \\ Sevilla, Spain. jcuevas@us.es \\ 2 Grupo de Física No Lineal, Departamento de Física Aplicada I, Escuela Técnica \\ Superior de Ingeniería Informática. Universidad de Sevilla. Avda. Reina \\ Mercedes, s/n. 41012 Sevilla, Spain. palmero@us.es
}

\section{Introduction}

The past few years have witnessed an explosion of interest in discrete models and intrinsic localized modes (discrete breathers or solitons) that has been summarized in a number of recent reviews [1]. This growth has been motivated by numerous applications of nonlinear dynamical lattice models in areas as broad and diverse as the nonlinear optics of waveguide arrays [2], the dynamics of Bose-Einstein condensates in periodic potentials [3], micro-mechanical models of cantilever arrays [4], or even simple models of the complex dynamics of the DNA double strand [5]. Arguably, the most prototypical model among the ones that emerge in these settings is the Discrete Nonlinear Schrödinger (DNLS) equation, the main topic of this book.

While DNLS combines two important features of many physical lattice systems, namely nonlinearity and periodicity, yet another element which is often physically relevant and rather ubiquitous is disorder. Localized impurities are well-known in a variety of settings to introduce not only interesting wave scattering phenomena [6], but also to create the possibility for the excitation of impurity modes, which are spatially localized oscillatory states at the impurity sites [7]. Physical applications of such phenomena arise, e.g., in superconductors [8], in the dynamics of the electron-phonon interactions [9], in the propagation of light in dielectric super-lattices with embedded defect layers [10] or in defect modes arising in photonic crystals [11].

In the context of the DNLS, there has been a number of interesting studies in connection to the interplay of the localized modes with impurities. Some of the initial works were either at a quasi-continuum limit (where a variational approximation could also be implemented to examine this interplay) [12] or at a more discrete level but with an impurity in the coupling [13] (see also in the latter setting the more recent studies of a waveguide bend $[14,15]$ and the boundary defect case of Ref. [16]). More recently the experimental inves- 
tigations of Refs. [17, 18] motivated the examinations of linear and nonlinear defects in a DNLS context [19, 20, 21].

The aim of the present chapter is to summarize the properties of the focusing DNLS equation in the presence of a linear impurity, as shown previously in [22]. Our first aim is to present the full bifurcation diagram of the stationary localized modes in the presence of the impurity and how it is drastically modified in comparison to the case of the homogeneous lattice. The relevant bifurcations are quantified whenever possible even analytically, in good agreement with our full numerical computations. A second aim is to show the outcome of the interaction of an incoming solitary wave with the linear impurity.

The DNLS equation with the defect can be written as,

$$
i \dot{u}_{n}+\gamma\left|u_{n}\right|^{2} u_{n}+\epsilon\left(u_{n+1}+u_{n-1}\right)+\alpha_{n} u_{n}=0, \quad(n=1 \ldots N)
$$

$\alpha_{n}$ allow for the existence of local, linear inhomogeneities. Hereafter, we consider a single point defect, thus $\alpha_{n}=\alpha \delta_{n, 0}$, that can be positive (attractive impurity) or negative (repulsive impurity). In general, the presence of an onsite defect would affect the nearest neighbor coupling, and Eq. (1) should be modified to take this effect into account, as in Ref. [23]. This inhomogeneity in the coupling, however, can be avoided using different techniques, for example, in nonlinear waveguide arrays, changing slightly the separation between the defect waveguide and its nearest neighbors, as in the case of Ref. [24]. We will assume here that the coupling parameter $\epsilon$ is independent of the site and positive.

Note that the defocusing case can be reduced, under the staggering transformation $u_{n} \rightarrow(-1)^{n} u_{n}$, to the previous one with opposite sign of the impurity $\alpha$. Also, under the transformation $u_{n} \rightarrow u_{n} e^{2 i \epsilon t}$, Eq. (1) can be written in the standard form

$$
i \dot{u}_{n}+\left|u_{n}\right|^{2} u_{n}+C \Delta u_{n}+\alpha_{n} u_{n}=0,
$$

In what follows, we use the form given by Eq. (1).

\section{Stationary solutions}

In this part, we look for stationary solutions with frequency $\Lambda, u_{n}(t)=e^{i \Lambda t} v_{n}$ and the stationary analog of Eq. (1) then reads

$$
-\Lambda u_{n}+\epsilon\left(u_{n+1}+u_{n-1}\right)+u_{n}^{3}+\alpha_{n} u_{n}=0 .
$$

\subsection{Linear modes}

Some of the properties of solitons are related to the characteristics of linear localized modes. These modes arise when an inhomogeneity appears and can 
be obtained from the linearized form (around the trivial solution $u_{n}=0, \forall n$ ) of Eq. (3). In this case, and considering an inhomogeneity located at the first site of the chain and with periodic boundary conditions, the problem reduces to solving the eigenvalue problem

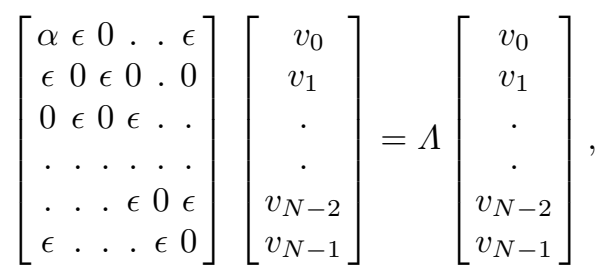

that is a particular case of the eigenvalue problem studied in Ref. [25]. There it was shown that, if $\alpha \neq 0$, the solution corresponds to $N-1$ extended modes and an impurity localized mode. Also, if $N$ becomes large, the frequencies of extended modes are densely distributed in the interval $\Lambda \in[-2 \epsilon, 2 \epsilon]$ and the localized mode can be approximated by

$$
v_{n}=s^{n} v_{0}\left[\left(\frac{\alpha}{2 \epsilon}+\beta\right)^{-n}+s^{N}\left(\frac{\alpha}{2 \epsilon}+\beta\right)^{n-N}\right], \quad \Lambda=2 s \epsilon \beta, \quad \beta \equiv \sqrt{\frac{\alpha^{2}}{4 \epsilon^{2}}+1}
$$

with $s=\operatorname{sign}(\alpha)$ and $v_{0}$ is an arbitrary constant. Note that for $\alpha>0(\alpha<0)$ the localized mode has an in-phase (staggered) pattern. In Fig. 1 we depict the linear mode spectrum as a function of the inhomogeneity parameter $\alpha$ and examples of the profiles of the ensuing localized modes.

(a)

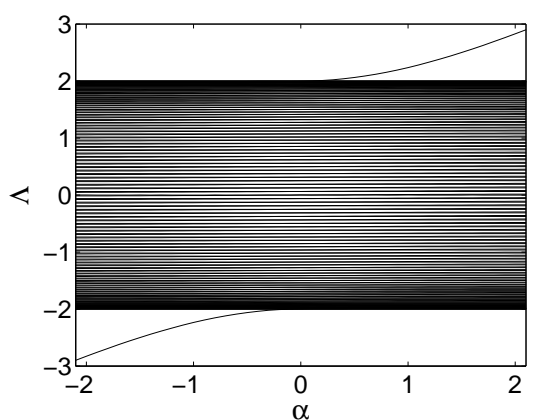

(b)
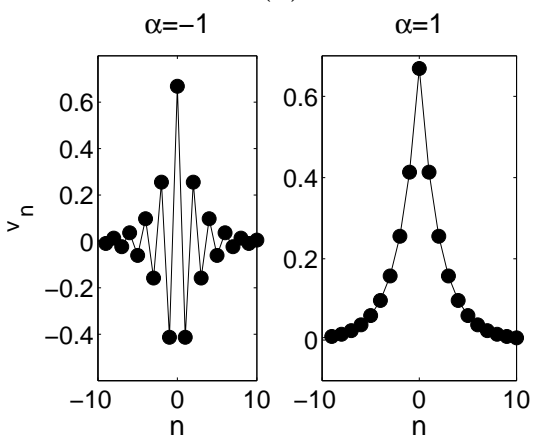

Fig. 1. (a) Linear modes spectrum as function of impurity parameter $\alpha$. Periodic boundary conditions are considered. (b) Examples of the profiles of the impurity modes. The impurity is located at $n=0$. (Left) profile for $\alpha=-1$; (right) profile for $\alpha=1$. In all cases $N=200$ and $\epsilon=1$. 


\subsection{Bifurcations}

In order to explore the existence and stability of the nonlinear stationary states described by Eq. (3), we have used the well-known technique based on the concept of continuation from the anti-continuum (AC) limit using a Newton-Raphson algorithm. Also, a standard linear stability analysis of these stationary states has been performed.

In the homogeneous lattice case of $\alpha=0$, fundamental stationary modes are well known to exist and be centered either on a lattice site or between two adjacent lattice sites. The site-centered solitary waves are always stable, while the inter-site centered ones are always unstable [26].
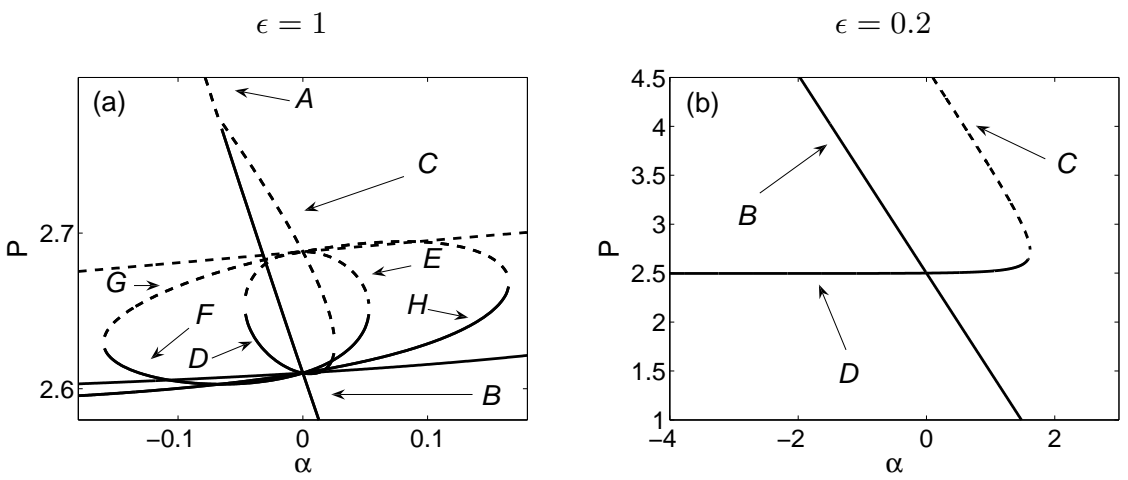

Fig. 2. Bifurcation diagram of stable (solid line) and unstable (dashed line) nonlinear modes. Shown is the power $P$ as a function of the impurity parameter $\alpha$. In all cases $N=100$ and $\Lambda=2.5$. The branch designation is as follows: (A) Unstable soliton centered at the impurity $(n=0),(\mathrm{B})$ stable on-site soliton centered at $n=0$, (C) Unstable inter-site soliton centered at $n=0.5$, (D) stable on-site soliton at $n=1,(\mathrm{E})$ unstable inter-site soliton at $n=1.5,(\mathrm{~F})$ stable on-site soliton at $n=2,(\mathrm{G})$ unstable inter-site soliton at $n=2.5$, and $(\mathrm{H})$ stable on-site soliton at $n=3$. The stable on-site mode located at the impurity, in the homogeneous case $(\alpha=0)$, disappears for a coupling value of $\epsilon \simeq 1.25$ due to resonances with the phonon band.

In order to study the effects of the inhomogeneity on the existence and properties of localized modes, we have performed a continuation from the homogeneous lattice case of $\alpha=0$. We found that, if $\alpha$ increases, $(\alpha>0$, attractive impurity case), the amplitude of the stable on-site mode decreases, while if $\alpha$ decreases ( $\alpha<0$, repulsive impurity case), in general, the stable on-site soliton localized at the impurity merges with the unstable inter-site centered one localized between the impurity and its neighboring site (beyond some critical value of $|\alpha|)$ and the resulting state becomes unstable. Notice that, at heart, the latter effect is a pitchfork bifurcation as the on-site mode 
collides with both the inter-site mode centered to its right, as well as with the one centered to its left.

In Fig. 2 we show a typical bifurcation scenario where, for fixed values of $\Lambda$ and $\epsilon$, we depict the mode power $P$ corresponding to different on-site and inter-site localized modes as a function of impurity parameter $\alpha$. If we denote as $n_{0}$ the site of the impurity, when $\alpha>0$ increases, we found that the unstable intersite soliton localized at $n=0.5$ disappears in a saddlenode bifurcation with the stable on-site soliton localized at $n=1$. Also, if we continue this stable mode, when $\alpha$ decreases, and for a given value $\alpha=\alpha_{c}<0$, it also disappears together with the unstable mode localized at $n=1.5$ through a saddle-node bifurcation. If we increase again the impurity parameter, this unstable mode localized at $n=1.5$ bifurcates with the stable site mode localized at $n=2$ for a critical value of parameter $\alpha=\alpha_{c}^{\prime}>0$ through a saddle-node bifurcation again, and it could be possible to continue this bifurcation pattern until a site $k$, where the value of site $k$ increases with the value of $\epsilon$ and $\Lambda$ parameters. This scenario is similar to the one found in previous studies with different kinds of impurities [14, 20] and appears to be quite general. It should be noted that when the coupling parameter increases, more bifurcations take place, in a narrower interval of power $P$ and impurity parameter $\alpha$ values.

Some of the particularly interesting experimentally tractable suggestions that this bifurcation picture brings forward are the following:

- A localized mode centered at the impurity may be impossible for sufficiently large attractive impurities (because the amplitude of the mode may decrease to zero), while it may be impossible to observe also in the defocusing case due to the instability induced by the pitchfork bifurcation with its neighboring inter-site configurations.

- A localized on-site mode centered at the neighborhood of the impurity should not be possible to localize for sufficiently large impurity strength both in the attractive and in the repulsive impurity case.

\subsection{Invariant manifold approximation}

This subsection shows a more detailed study of the bifurcation between the on-site nonlinear mode centered at the impurity and its inter-site and one-site neighbor. From the discussion of the previous section we can determine for a given value of the coupling parameter $\epsilon$, the corresponding critical value of impurity parameter $\alpha=\alpha_{c}$. Note that this bifurcation takes place only if $\alpha$ is negative (repulsive impurity). In case of $\alpha$ positive (attractive impurity), the inter-site solution disappears in a saddle-node bifurcation with the on-site wave centered at the site next to the impurity at $\alpha=\alpha_{c}^{\prime}$. In these cases, via an analysis of invariant manifolds of the DNLS map, and following the method developed in Section 4.1.4 of Ref. [27], some approximate analytical expressions corresponding to this bifurcation point can be obtained. This method is sketched below. 
The difference equation (3), for $\alpha=0$, can be recast as a two-dimensional real map by defining $y_{n}=v_{n}$ and $x_{n}=v_{n-1}[28]$ :

$$
\left\{\begin{array}{l}
x_{n+1}=y_{n} \\
y_{n+1}=\left(\Lambda y_{n}-y_{n}^{3}\right) / \epsilon-x_{n} .
\end{array}\right.
$$

For $\Lambda>2$, the origin $x_{n}=y_{n}=0$ is hyperbolic and a saddle point. Consequently, there exists a one-dimensional stable $\left(W^{s}(0)\right)$ and a one-dimensional unstable $\left(W^{u}(0)\right)$ manifolds emanating from the origin in two directions given by $y=\lambda_{ \pm} x$, with

$$
\lambda_{ \pm}=\frac{\Lambda \pm \sqrt{\Lambda^{2}-4 \epsilon^{2}}}{2 \epsilon} .
$$

These manifolds intersect in general transversally, yielding the existence of an infinity of homoclinic orbits. Each of their intersections corresponds to a localized solution. Fundamental solitons (i.e. on-site and inter-site solitons), correspond to the primary intersection points, i.e. those emanating from the first homoclinic windings. Each intersection point defines an initial condition $\left(x_{0}, y_{0}\right)$, that is, $\left(v_{-1}, v_{0}\right)$, and the rest of the points composing the soliton are determined by application of the map (6) and its inverse. Fig. 3 shows an example of the first windings of the manifolds. Intersections corresponding to fundamental solitons are labeled as follows: (1) is the on-site soliton centred at $n=0,(2)$ is the inter-site soliton centred at $n=0.5$ and (3) is the on-site soliton centred at $n=1$.

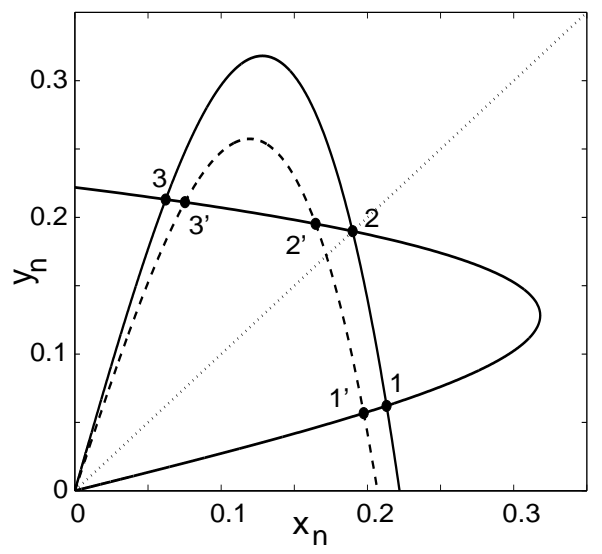

Fig. 3. First winding of the homoclinic tangle of the map (6). Dashed line corresponds to the linear transformed unstable manifold when $\alpha=0$. Labels $1,2,3\left(1^{\prime}\right.$, $\left.2^{\prime}, 3^{\prime}\right)$ corresponds to fundamental solitons for $\alpha=0(\alpha \neq 0)$.

The effect of the inhomogeneity is introduced as a linear transformation of the unstable manifold $A(\alpha) W^{u}(0)$ with $A(\alpha)$ given by: 


$$
A(\alpha)=\left[\begin{array}{cr}
1 & 0 \\
-\alpha / C & 1
\end{array}\right]
$$

When $\alpha>0$, the unstable manifold moves downwards, changing the intersections between the transformed unstable manifold and the stable manifold to points $1^{\prime}, 2^{\prime}$ and $3^{\prime}$ (see Fig. 3). For $\alpha=\alpha_{c}$, both manifolds become tangent. Thus, for $\alpha>\alpha_{c}^{\prime}$ intersections $3^{\prime}$ and $2^{\prime}$ are lost, that is, for $\alpha=\alpha_{c}^{\prime}$ the breathers centred at $n=1$ and $n=0.5$ experience a tangent bifurcation. On the contrary, if $\alpha<0$, intersections $1^{\prime}$ and $2^{\prime}$ are lost when $|\alpha|>\left|\alpha_{c}\right|$, leading to a bifurcation between the breathers centered at $n=0.5$ and $n=0$.

A method for estimating $\alpha_{c}(\Lambda)$ and $\alpha_{c}^{\prime}(\Lambda)$ is based on a simple approximation of $W^{u}(0)$. Let us consider a cubic approximation $W_{\text {app }}^{u}$ of the local unstable manifold of Fig. 3, parametrized by $y=\lambda x-c^{2} x^{3}$, with $\lambda \equiv \lambda_{+}$. The coefficient $c$ depends on $\Lambda$ and $C$ and need not be specified in what follows (a value of $c$ suitable when $\lambda$ is large is computed in Ref. [29]). We have

$$
y=\lambda_{0} x-c^{2} x^{3}
$$

on the curve $A(\alpha) W_{\text {app }}^{u}$, where $\lambda_{0}=\lambda-\alpha / C$. By symmetry we can approximate the local stable manifold using the curve $W_{\text {app }}^{s}$ parametrized by

$$
x=\lambda y-c^{2} y^{3} .
$$

The curves $A(\alpha) W_{\text {app }}^{u}$ and $W_{\text {app }}^{s}$ become tangent at $(x, y)$ when in addition

$$
\left(\lambda-3 c^{2} x^{2}\right)\left(\lambda_{0}-3 c^{2} y^{2}\right)=1 .
$$

In order to compute $\alpha_{c}$ and $\alpha_{c}^{\prime}$ as a function of $\Lambda$, or, equivalently, the corresponding value of $\lambda_{0}$ as a function of $\lambda$, one has to solve the nonlinear system (9)- (11) with respect to $x, y, \lambda_{0}$, which yields a solution depending on $\lambda$. Instead of using $\lambda$ it is practical to parametrize the solutions by $t=y / x$. This yields

$x=\frac{1}{c \sqrt{2}}\left(t+\frac{1}{t^{3}}\right)^{1 / 2}, \quad y=\frac{t}{c \sqrt{2}}\left(t+\frac{1}{t^{3}}\right)^{1 / 2} ; \quad \lambda_{0}=\frac{3}{2} t+\frac{1}{2 t^{3}}, \quad \lambda=\frac{3}{2 t}+\frac{1}{2} t^{3}$.

Since $\lambda+\lambda^{-1}=\Lambda / \epsilon$ it follows that

$$
\begin{gathered}
t^{4}-2 \lambda t+3=0, \\
\alpha=\frac{\epsilon}{2}\left(t-\frac{1}{t}\right)^{3} .
\end{gathered}
$$

As this system of equations has two real positive solutions, given a value of $\Lambda$, one can approximate $\alpha_{c}$ and $\alpha_{c}^{\prime}$ by the values of $\alpha$ given by equations (12)-(13). Despite the fact that it gives precise numerical results in a certain parameter range, the approximation (12)-(13) is not always valid. Indeed, the 
parameter regime $\Lambda<5 \epsilon / 2$ is not described within this approximation (see [29]).

Fig. 4 shows the comparison between the exact numerical and the approximate analytical results. For a fixed value of the coupling parameter $\epsilon$, the critical value of the frequency increases with $|\alpha|$.
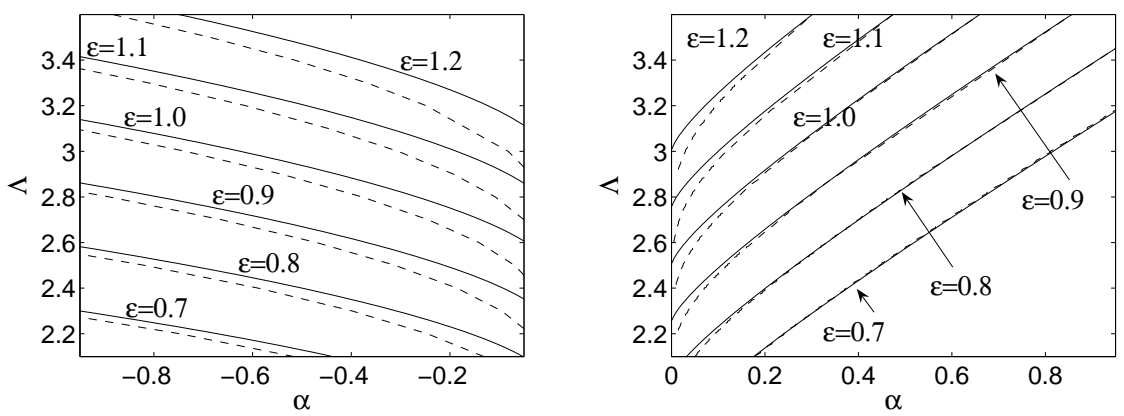

Fig. 4. Bifurcation loci corresponding to the bifurcation between the on-site localized mode at the impurity $(n=0)$ and its neighbor inter-site breather $(n=0.5)$ (left panel), and to the bifurcation between the on-site localized mode next to the impurity $(n=1)$ and its neighbor inter-site breather $(n=0.5)$ (right panel), for different values of parameter $\epsilon$. Dashed lines correspond to numerical results and continuous lines to approximate analytical calculations.

\section{Interaction of a moving soliton with a single impurity}

Early studies of the DNLS had shown that discrete solitary waves in the DNLS can propagate along the lattice with a relatively small loss of energy [30], and more recent work suggests that such (almost freely) propagating solutions might exist, at least for some range of control parameters [31, 32, 33]; nevertheless, genuinely traveling single-hump solitary wave solutions are not present in the DNLS, but only in variants of that model such as the ones with saturable nonlinearity [34].

In this section we deal with the interaction of propagating (with only weak radiative losses) localized modes with the impurity. Thus, we consider a nonlinear localized mode, far enough from the impurity, of frequency $\Lambda$, and perturb it by adding a thrust $q$ to a stationary breather $v_{n}$ [35], so that:

$$
u_{n}(t=0)=v_{n} e^{i q n} .
$$

This is similar in spirit to the examination of Ref. [19], although we presently examine both attractive and repulsive impurities. In what follows, $\Lambda=2.5$ and $\epsilon=1$; a similar scenario emerges for other values of $\Lambda$. 
In general, if $q$ is large enough, the soliton moves with a small loss of radiation. We have calculated, as a function of parameters $q$ and $\alpha$, the power and energy that remains trapped by the impurity, reflected and transmitted along the chain, and determined the corresponding coefficients of trapping, reflection and transmission, defined as the corresponding fraction of power (energy). Fig. 5 summarizes the relevant numerical results.
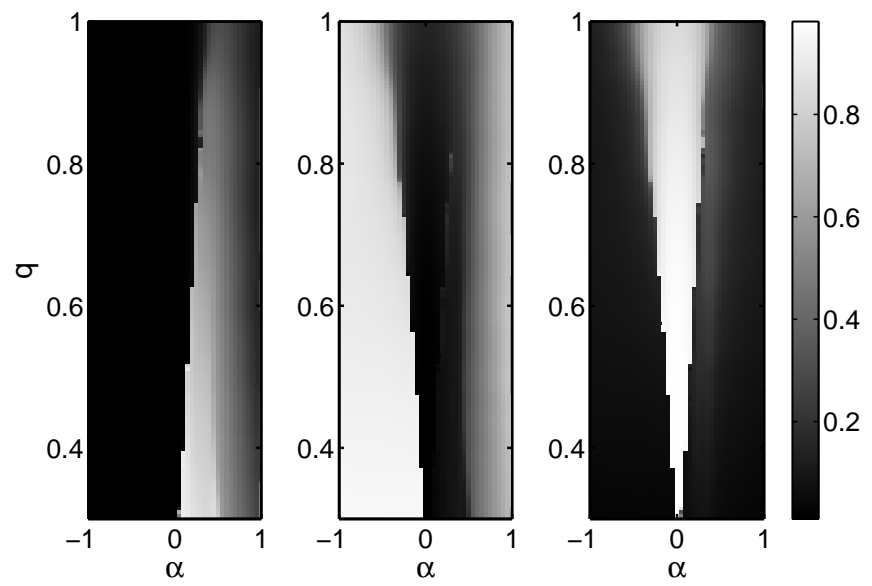

Fig. 5. Power trapping (left), reflection (center) and transmission (right) coefficients as function of impurity parameter $\alpha$ and initial thrust $q$. In all cases $\epsilon=1$.

We can essentially distinguish four fundamental regimes:

(a) Trapping. If parameters $q$ and $\alpha$ are small enough, and the impurity is attractive, nearly all the energy remains trapped at the impurity, and only a small fraction of energy is lost by means of phonon radiation. An example of this phenomenon is shown in Fig. 6 (left). In this case, the central power (power around the impurity) before the collision is nearly zero. When the localized mode reaches the impurity, the former loses power as phonon radiation and remains trapped. The analysis of the Fourier spectrum of this trapped breather, carried out after the initial decay and at an early stage of the evolution, shows a frequency close to the initial soliton frequency, as shown in Fig. 6 (right). We have observed that, in general, this frequency is slightly smaller than that of the incident soliton, and, in consequence, it has even smaller energy (in absolute value) and power than the corresponding nonlinear mode with the frequency of incident soliton. In this particular case, corresponding to $q=0.3$ and $\alpha=0.2$, the initial incident wave (after perturbation) has power $P=2.61$ and energy $E=-5.40$ and the stationary mode, trapped at the impurity, with the same frequency, has 
$P=2.17$ and $E=-4.73$. Thus, the incident breather can activate this nonlinear mode, and nearly all energy and norm remains trapped.
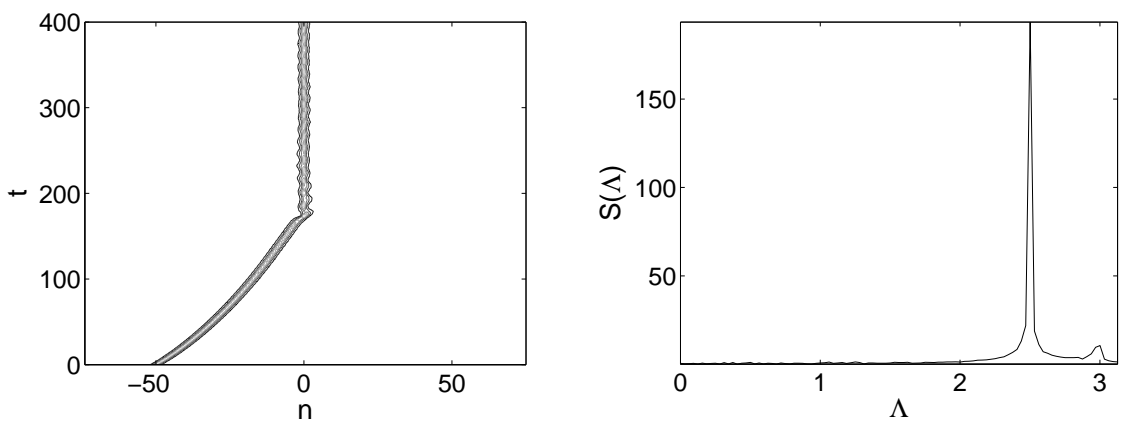

Fig. 6. Trapping: Contour plot corresponding to the power of the soliton $P$ as function of site $n$ and time $t$ (left panel) and Fourier power spectrum of the trapped soliton calculated soon after the collision (right panel). The parameters are $\alpha=0.2$, $q=0.3, \Lambda=2.5, \epsilon=1$ and the impurity is located at $n=0$.

(b) Trapping and reflection. If the impurity is attractive, but strong enough, some fraction of energy remains trapped by the impurity, but a considerable amount of it is reflected. The reflected excitation remains localized. This case is similar to the previous one, but now the incident traveling structure has enough energy and norm to excite a stationary mode centered at the impurity, remaining localized and giving rise to a reflected pulse. A typical case is shown in Fig. 7, that corresponds to $q=0.6$ and $\alpha=1.0$. The incident wave has power and energy $P=2.61$ and $E=-4.79$, and the stationary nonlinear mode centered at the impurity, with the same frequency, $P=0.76$ and $E=-1.79$. When the incident breather reaches the impurity, it excites the nonlinear mode, and, after losing some power, part of it remains localized, and another part is reflected. Also, in our numerical simulations, we have detected, as in the previous case, that the frequency of the remaining trapped mode is slightly lower than that the incident breather, so it has even smaller power than the corresponding nonlinear mode with the frequency of incident soliton.

In general, we have found that a necessary condition to trap energy and power by the impurity is the existence of a nonlinear localized mode centered at the impurity, with similar frequency, and energy (in absolute value) and power smaller than that of the corresponding incident soliton.

(c) Reflection with no trapping. Here, we have to distinguish two cases. If the impurity is repulsive, and $q$ small enough, neither trapping, nor transmission occur. Instead, all energy is reflected, and the traveling nonlinear excitation remains localized. In this case, as shown in Fig. 8 (left), the 

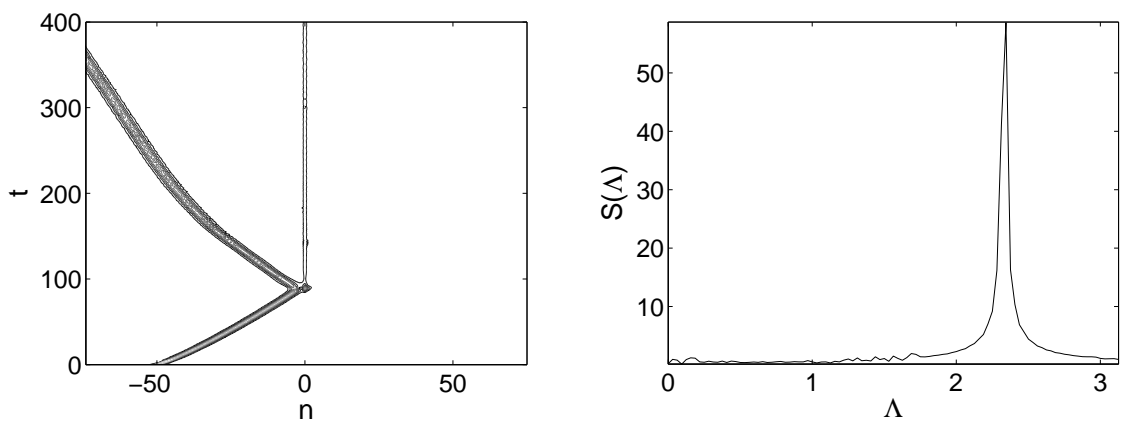

Fig. 7. Trapping and reflection: Contour plot corresponding to the power of soliton $P$ as a function of site $n$ and time $t$ (left panel) and Fourier power spectrum of the trapped soliton calculated soon after the collision (right panel). The parameters are $\alpha=1.0, q=0.6, \Lambda=2.5, \epsilon=1$ and the impurity is located at $n=0$.
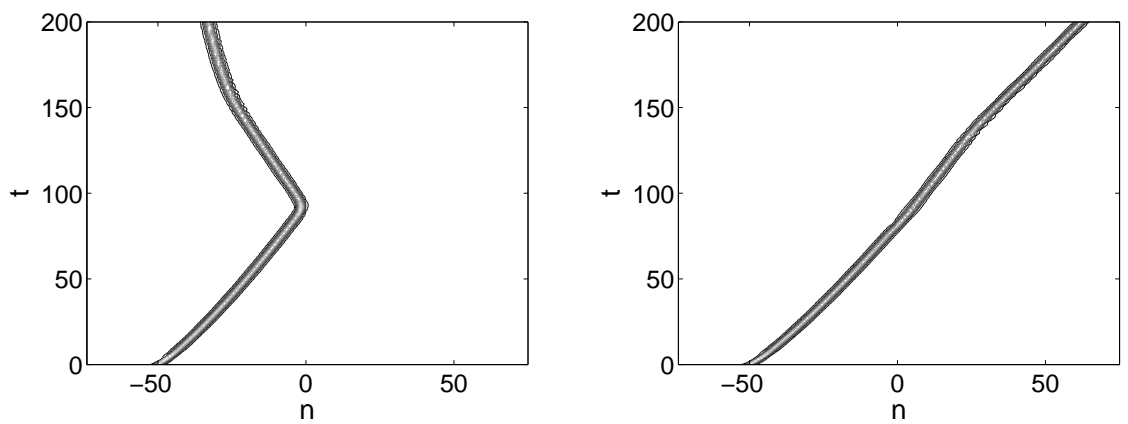

Fig. 8. Reflection with no trapping (left panel) corresponding to parameters $\alpha=-0.5, q=0.6$ and $\Lambda=2.5$ and transmission with no trapping (right panel) corresponding to parameters $\alpha=0.1, q=0.7$ and $\Lambda=2.5$. In both cases we represent a contour plot corresponding to the power of the soliton $P$ as function of site $n$ and time $t, \epsilon=1$ and the impurity is located at $n=0$.

incident wave has no energy and power to excite the localized mode. In a typical case, i.e., $\Lambda=2.5, q=0.6$ and $\alpha=-0.5$, the incident soliton has energy and power $E=-4.79$ and $P=2.61$, and the nonlinear localized mode on the impurity with the same frequency $E=-8.038$ and $P=3.77$. No trapping phenomenon occurs, and the pulse is reflected.

On the other hand, if the impurity is attractive and strong enough, i.e., $q=0.7, \Lambda=2.5$ and $\alpha=2.0$, the frequency of the soliton is smaller than the one corresponding to the linear impurity mode $\left(\Lambda_{L} \simeq 2.82\right)$, and all the energy is reflected. This is in accordance with the necessity of a nonlinear localized mode at the impurity site in order for the trapping to occur. 

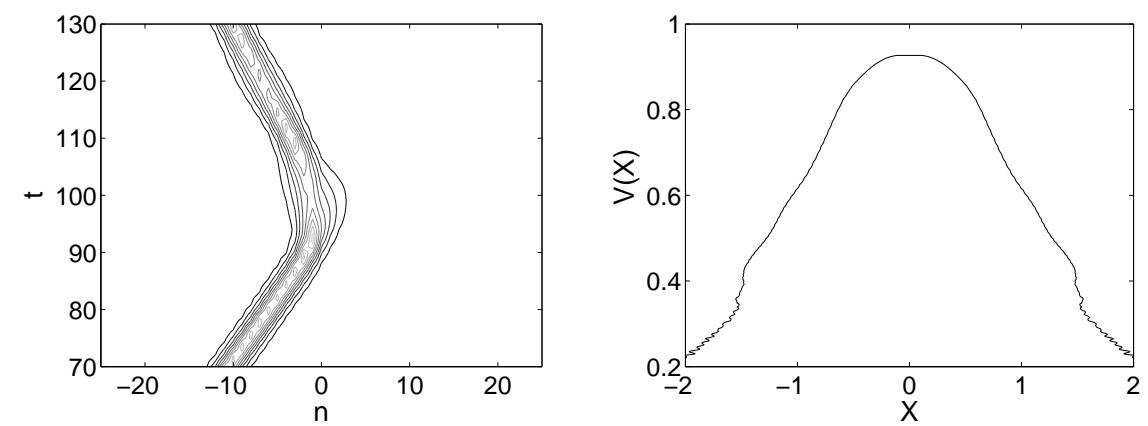

Fig. 9. Contour plot of the phenomenon of reflection of a soliton corresponding to thrust parameter $q=0.6$ (left panel). Potential barrier calculated as described in text (right panel). In both cases $\alpha=-0.2, \epsilon=1, \Lambda=2.5$ and the impurity is located at $n=0$.

(d) Transmission with no trapping. If $|\alpha|$ is small enough, and $q$ high enough, transmission with no trapping occurs, as shown in Fig. 8 (right). There exists a critical value of $q=q_{c}>0$ such that, if $q>q_{c}$, the incident soliton crosses through the impurity. The value of $q_{c}$ grows with $|\alpha|$. In the case where $q<q_{c}$, if $\alpha<0$, reflection with no trapping occurs, while if $\alpha>0$, trapping with no reflection phenomenon takes place.

Our results related to trapping, reflection and transmission phenomena are in agreement with some results recently obtained, using a different approach, in a similar system [19]. In this work, where approximate discrete moving solitons with fixed amplitude are generated using a continuous approximation, the authors study the trapping process by a linear and a nonlinear attractive impurity. In the former framework, trapping can be explained by means of resonances with the linear localized mode. In our case, where nonlinear effects become stronger, the phenomena are related to resonances with a nonlinear localized mode.

Finally, a very interesting phenomenon occurs when the parameter $\alpha$ is repulsive and small enough (in absolute value). In this case, the solitary wave can be reflected or transmitted depending on its velocity. Also, when it is reflected, our numerical tests show that its velocity is similar to its incident velocity. Thus, if we consider the soliton as a "quasiparticle", the effect of the impurity is similar to the effect of a potential barrier. To determine this potential barrier for a given value of parameter $\alpha$, we have used a method similar to the one described by Ref. [36]. We have considered different values of the thrust parameter $q$ corresponding to the reflection regime, and determine, for each value, the turning point, $X(q)$. Thus the translational energy of the barrier for this value of $q$ is defined as the difference between the energy of the moving soliton and the stationary state of the same frequency far from 
the impurity. It can be written as $V(q)=C \sin (q / 2)|P(q / 2)|$, with $P(q)=$ $i \sum_{n} \psi_{n}^{*} \psi_{n+1}-\psi_{n}^{*} \psi_{n-1}$ being the lattice momentum, as defined in Ref. [37]. Results are shown in Fig. 9, which exhibits, as expected, an irregular shape, whose origin lies in the nonuniform behavior of the translational velocity due to the discreteness of the system.

On the other hand, if the parameter $\alpha$ is small enough, and positive (attractive), the solitary wave faces a potential "well" and can be trapped if its translational energy is small or, if the translational energy is high enough, it may be transmitted, losing energy that remains trapped by the impurity, and decreasing its velocity.

To sum up, we have examined in detail for both impurity cases (attractive and repulsive) the interaction of the impurity with a moving localized mode initiated away from it. The principal regimes that we have identified as a function of the impurity strength (and sign) and initial speed are trapping, partial trapping and partial reflection, pure reflection and pure transmission. In general, if the impurity is repulsive, and the speed small enough, the wave is always reflected. If the impurity strength (in absolute value) is small enough and the speed is high enough, then transmission can take place. On the other hand, if the impurity is attractive, trapping can occur, and if the speed is high enough two different effects are observe: for small values of $\alpha$, transmission takes place and for high values of $\alpha$, the trapping is accompanied by a partial reflection (for intermediate values of $\alpha$, the trapping is pure). If the impurity is attractive and sufficiently strong, the frequency of the soliton is smaller than the one corresponding to the linear localized impurity mode and the wave is reflected.

The above described scenario is slightly different to the shown in [19], where small-amplitude solitons are considered. Contrary to the high-amplitude solitons considered in [22] where the trapping was shown to be originated by the excitation of a nonlinear localized mode centred at the impurity site, in [19], the trapping is due to excitations of linear localized modes, as the frequency of small-amplitude solitons are close to the phonon band. Thus, the scenario observed for attractive impurities is the following: for small impurity strength, the soliton is transmitted; above a critical value of $\alpha$, the soliton is trapped partially, with the reflected fraction increasing with the impurity strength.

\section{Comparison with other related models}

The findings herein, while presented for a linear impurity are representative of other models including DNLS lattices with nonlinear impurities and KleinGordon lattices. We briefly expose hereafter the main similarities and differences between them. 


\subsection{Nonlinear impurities}

Stationary solitons with a quintic nonlinear impurity were considered in [20]:

$$
i \dot{u}_{n}+\left|u_{n}\right|^{2} u_{n}+\epsilon\left(u_{n+1}+u_{n-1}-2 u_{n}\right)+\alpha \delta_{n, 0}\left|u_{n}\right|^{4} u_{n}=0,
$$

In that work, only attractive impurities were considered. The bifurcation diagram of solitons close to the impurity site is similar to the one found for linear impurities (see Fig. 2). By means of a variational approximation the value of the impurity strength at which the branches corresponding to solitons centred at $n=0$ and $n=0.5$ merge. The dependence of this critical value with the frequency is:

$$
\alpha_{c}(\Lambda)=-\frac{16 \pi^{4} \epsilon^{3}}{\Lambda^{4}} \exp \left(-\pi^{2} \sqrt{\frac{\epsilon}{\Lambda}}\right) .
$$

This dependence was approximated in the linear impurity case by means of an invariant manifold approximation (see Section 2.3).

The interaction of small-amplitude moving solitons with nonlinear impurities has been briefly considered in [19], where, contrary to (15), a nonlinear cubic impurity was considered:

$$
i \dot{u}_{n}+\left(1+\alpha \delta_{n, 0}\right)\left|u_{n}\right|^{2} u_{n}+\epsilon\left(u_{n+1}+u_{n-1}\right)=0 .
$$

The observed scenario for attractive impurities was the following: for small $\alpha$, the soliton is transmitted and, above a threshold value, the soliton is trapped without reflection. If the impurity is increased, the soliton is totally reflected, and, surprisingly, for relatively high values of $\alpha$, the soliton is trapped again.

\subsection{Comparison with Klein-Gordon breathers}

The interaction of moving localized in a Klein-Gordon (KG) chains (called discrete breathers) with a point inhomogeneity in the substrate potential was considered in [38]. The model equation is given by:

$$
\ddot{u}_{n}+V_{n}^{\prime}\left(u_{n}\right)+C\left(2 u_{n}-u_{n+1}-u_{n-1}\right)=0
$$

with $V\left(u_{n}\right)=\left(1+\alpha \delta_{n, 0}\right)\left(\exp \left(-u_{n}\right)-1\right)^{2} / 2$ being the Morse substrate potential (for different potentials or kinds of impurities, the reader is referred to [39]). In this setting, for $\alpha>0(\alpha<0)$ the impurity is repulsive (attractive) contrary to the DNLS case shown throughtout the present chapter.

The observed regimes for KG breathers are qualitatively equivalent to those of DNLS solitons. An important analogy in both settings is the necessary condition for the trapping; i.e. the energy of the moving localized mode must be higher (in absolute value) than that of the stationary localized mode centred at the impurity (with the same frequency of the moving soliton/breather). This result for Klein-Gordon lattices was established in [38, 40]. 
It is worth mentioning the study performed in [27] where many existence conditions are established for stationary Klein-Gordon breathers in inhomogeneous lattices based on a centre manifold approach. The latter work also predicted and illustrated gap breathers, that is breathers whose frequency lies in the gap left in the phonon band by the linear localized mode when depart from it. These structures, however, do not exist in DNLS lattices.

\section{Summary and future challenges}

In the present chapter, the existence and stability of discrete solitons close to a local inhomogeneity in a 1-D DNLS lattice have been studied. A systematic study of the interaction of a moving discrete soliton with that local inhomogeneity has been performed. Finally, a brief comparison of these results with other related settings, such as DNLS lattices with nonlinear impurities or Klein-Gordon lattices, was given.

Further development of this direction of research could include the consideration of saturable nonlinearities which can describe nonlinear waveguides made of photorefractive materials. This kind of nonlinearity may enhance the mobility of solitary waves in isotropic two-dimensional lattices [41], whereas moving solitons only can take place in anisotropic 2D lattices for cubic lattices [42]. A similar study to that shown in the present section could be done in both settings. Another interesting direction could be the inclusion of two or more local inhomogeneities and the examination of the interplay between them.

\section{References}

1. S. Aubry, Physica D 103, 201, (1997); S. Flach and C.R. Willis, Phys. Rep. 295, 181 (1998); D. Hennig and G. Tsironis, Phys. Rep. 307, 333 (1999);

2. D. N. Christodoulides, F. Lederer and Y. Silberberg, Nature 424, 817 (2003);

3. O. Morsch and M. Oberthaler, Rev. Mod. Phys. 78, 179 (2006); P.G. Kevrekidis and D.J. Frantzeskakis, Mod. Phys. Lett. B 18, 173 (2004).

4. M. Sato, B. E. Hubbard, and A. J. Sievers, Rev. Mod. Phys. 78, 137 (2006).

5. M. Peyrard, Nonlinearity 17, R1 (2004).

6. A.A. Maradudin, Theoretical and Experimental Aspects of the Effects of Point Defects and Disorder on the Vibrations of Crystal (Academic Press, New York, 1966).

7. I.M. Lifschitz, Nuovo Cimento, Suppl. 3, 716 (1956); I.M. Lifschitz and A.M. Kosevich, Rep. Progr. Phys. 29, 217 (1966).

8. A.F. Andreev, JETP Lett. 46, 584 (1987); A.V. Balatsky, Nature (London) 403 717 (2000).

9. M.I. Molina and G.P. Tsironis, Phys. Rev. B 47, 15330 (1993); G.P. Tsironis, M.I. Molina, and D. Hennig, Phys. Rev. E 50, 2365 (1994).

10. E. Lidorikis, K. Busch, Q. Li, C.T. Chan, and C.M. Soukoulis, Phys. Rev. B 56, 15090 (1997). 
11. S.Y. Jin, E. Chow, V. Hietala, P.R. Villeneuve, and J.D. Joannopoulos, Science 282, 274 (1998); M.G. Khazhinsky and A.R. McGurn, Phys. Lett. A 237, 175 (1998).

12. K. Forinash, M. Peyrard, and B. Malomed, Phys. Rev. E 49, 3400 (1994).

13. W. Krolikowski and Yu.S. Kivshar, J. Opt. Soc. Am. B 13, 876 (1996).

14. Yu.S. Kivshar, P.G. Kevrekidis and S. Takeno, Phys. Lett. A 307, 287 (2003).

15. M. Agrotis, P.G. Kevrekidis and B.A. Malomed, Math. Comp. Simul. 69, 223 (2005).

16. S. Longhi, Phys. Rev. E 74, 026602 (2006).

17. U. Peschel, R. Morandotti, J.S. Aitchison, H.S. Eisenberg, and Y. Silberberg, Appl. Phys. Lett., 75, 1348 (1999).

18. R. Morandotti, H. S. Eisenberg, D. Mandelik, Y. Silberberg, D. Modotto, M. Sorel, C. R. Stanley, and J. S. Aitchison, Opt. Lett. 28, 834 (2003).

19. L. Morales-Molina, R.A. Vicencio, Opt. Lett. 31, 966 (2006).

20. P.G. Kevrekidis, Yu.S. Kivshar and A.S. Kovalev, Phys. Rev. E 67, 046604 (2003).

21. E. Smirnov, C.E. Rüter, M. Stepić, V. Shandarov and D. Kip, Opt. Express 14, $11248(2006)$.

22. F. Palmero, R. Carretero-González, J. Cuevas, P.G. Kevrekidis and W. Królikowski. Phys. Rev. E 77, 036614 (2008).

23. H. Trompeter, U. Peschel, T. Pertsch, F. Lederer, U. Streppel, D. Michaelis and A. Bräuer. Opt. Express 11, 3404 (2003).

24. R. Morandotti, U. Peschel, J.S. Aitchison, H.S. Eisenberg and Y. Silberberg, Phys. Rev. Lett. 83, 2726 (1999).

25. F. Palmero, J. Dorignac, J.C. Eilbeck and R.A. Römer. Phys. Rev. B 72, 075343 (2005).

26. P.G. Kevrekidis, K.Ø. Rasmussen, and A.R.

27. G. James, B. Sánchez-Rey and J. Cuevas. Preprint. arXiv:0710.4114

28. W.X. Qin and X.Xiao. Nonlinearity 20, 2305 (2007).

29. J. Cuevas, G. James, B.A. Malomed, P.G. Kevrekidis and B. Sánchez-Rey. Preprint. arXiv:0712.1837

30. J.C. Eilbeck. in: C. Kawabata, A.R. Bishop (Eds.), Computer Analysis for Life Science Progress and Challenges in Biological and Synthetic Polymer Research, Ohmsha, Tokyo, pp. 12-21 (1986).

31. H. Feddersen, in: M. Remoissenet, M. Peyrard (Eds.), Nonlinear Coherent Structures in Physics and Biology, in: Lecture Notes in Physics, vol. 393, Springer, pp. 159-167 (1991).

32. J.C. Eilbeck and M. Johansson, in: L. Vázquez, R.S. MacKay, M.P. Zorzano (Eds.), Localization and Energy Transfer in Nonlinear Systems, World Scientific, Singapore, pp. 44-67 (2003).

33. J. Gómez-Gardeñes, L.M. Floría, M. Peyrard and A.R. Bishop, Chaos 14, 1130 (2004).

34. T.R.O. Melvin, A.R. Champneys, P.G. Kevrekidis and J. Cuevas, Phys. Rev. Lett. 97, 124101 (2006); O.F. Oxtoby and I.V. Barashenkov, Phys. Rev. E 76, 036603 (2007); T.R.O. Melvin, A.R. Champneys, P.G. Kevrekidis and J. Cuevas, Physica D, 237, 551 (2008).

35. J. Cuevas and J.C. Eilbeck, Phys. Lett. A 358, 15 (2006).

36. J. Cuevas and P.G. Kevrekidis, Phys. Rev. E 69, 056609 (2004).

37. I.E. Papacharalampous, P.G. Kevrekidis, B.A. Malomed and D.J. Frantzeskakis 68, 046604 (2003). 
38. J. Cuevas, F. Palmero, J.F.R. Archilla and F. Romero. Journal of Physics A 35, 10519 (2002); J. Cuevas, F. Palmero, J.F.R. Archilla and F. Romero, Theor. Math. Phys. 137, 1406 (2003).

39. J. Cuevas. PhD Dissertation. University of Sevilla.

40. A. Alvarez, F.R. Romero, J.F.R. Archilla, J. Cuevas and P.V. Larsen, Eur. Phys. J. B 51, 119 (2006).

41. R. Vicencio and M. Johansson, Phys. Rev. E 73, 046602 (2006).

42. J. Gómez-Gardeñes, L.M. Floría and A.R. Bishop, Physica D 216, 31 (2006). 